\title{
Enhance and Compress the Dark Regions Depending on an Experimental Psychologist Law
}

\author{
Maha A. Hameed \\ Dept. of Astronomy, College of Science, University of Baghdad
}

\begin{abstract}
This paper combines the enhance poor light image (which has reducing contrast for dark regions in image) then compresses it by recognition a background regions. At first, A Block Analysis for Gray level images method has been used to detect the background region in image by depending on an information from image block with dark regions or poor lighting. The presented work has been carried out by two steps; The first step, an information from each image block has been analyzes by A Block Analysis for Gray level images method (i.e. background analysis based on high and low means for each block), while the second step, background for image with dark regions which has been enhanced is compressed. The aim of this paper is to detect the background image and enhance the contrast in gray level image with poor or reduced lighting then compresses it in low bit rates with good reconstructed enhanced image quality.
\end{abstract}

Keywords: Contrast enhancement, high and low means, Image Background Analysis, An experimental psychologist law

\section{Introduction}

For the last years, a successful movement has been started towards the direction of the improvement of image processing methods [1,2]. Many research has been carried out to enhance poor light images. Image enhancement is a method that increases the visual contrast of an image by detection of regions (such as edges, peaks, and other statistical features which is chief importance in poor light vision) $[3,4]$. The aim of enhancing image include the improvement of the visibility of the different regions into image. One can see that the enhanced image is more vision image than the original [5]. In this paper, the advanced of proposed method is the textural information has been rendered visible in the enhanced image. Where the presented work has been carried out by two steps. The first step, an information from each image block (i.e. high and low means for each block) has been analyzes by A Block Analysis for Gray level images method to determine the criteria value $\alpha$, where $\alpha$ is used as a threshold between dark and clear region, while the second step, background for image with dark regions which has been enhanced is compressed based on the Weber's law [3,5]. In other word, this paper presents a new image coding enhanced algorithm using Weber-Fechner condition which is an efficient method for decreasing computational complexity and improving compression results in low bit rates with good reconstructed enhanced image quality. Finally the aim of this paper is to detect the background in image and enhance the contrast in gray level image which has poor or reduced lighting then compresses it in low bit rates with good quality.

\section{Image Background Analysis by Blocks}

Let $\boldsymbol{G}$ be the original image which is subdivided into $\boldsymbol{n}$ blocks. For each block, the minimum intensity $\boldsymbol{m}_{\mathrm{i}}$ and maximum intensity $\boldsymbol{M}_{\boldsymbol{i}}$ values ( i.e. high and low means for each block) are calculated [6], these values are used to find the background criteria $\boldsymbol{\alpha}$ by using this equation [3-5]:

$$
\alpha_{i}=\frac{m_{i}+M_{i}}{2} \quad \forall i=1,2, \ldots \ldots ., n
$$

Where $\boldsymbol{\alpha}$ is used as a threshold between dark $\boldsymbol{F}<=\boldsymbol{\alpha}$ and clear $\boldsymbol{F}>\boldsymbol{\alpha}(\boldsymbol{F}$ is intensity levels). Depending on the value of $\boldsymbol{\alpha}$ (the background parameter) which is decided for each analyzed block, the contrast enhancement is expressed as follows:

$$
R_{\alpha_{i}}(F)= \begin{cases}k_{i} \log (F+1)+m_{i} & F>\alpha_{i} \\ k_{i} \log (F+1)+M_{i} & F \leq \alpha_{i}\end{cases}
$$

While the background parameter is totally dependent on the background criteria $\boldsymbol{\alpha}$ value, therefore, for $\boldsymbol{F}<=\boldsymbol{\alpha}$, the background parameter takes the maximum intensity value $\boldsymbol{M}_{\boldsymbol{i}}$ within the analyzed block, otherwise takes the minimum intensity value $\boldsymbol{m}_{\boldsymbol{i}}$. To avoid in determination condition, unit was added to the logarithmic function $[4,5]$.

$$
k_{i}=\frac{255-d}{\log (256)}, \text { where } d=\left\{\begin{array}{lc}
m_{i} & F \leq \alpha_{i} \\
M_{i} & \text { Otherwise }
\end{array}\right.
$$

In the enhanced images, it can be seen that the objects which are dark visible region in the original image are appeared.

\section{Contrast enhancement}

Digital image enhancement includes the improvement of the visibility and perceptibility of the different regions, which an image can be partitioned then defect ability of the image texture inside the regions. Enhanced image is usually followed by detection of features like edges, peaks, and other geometric features. One can see that the enhanced image is a sharper than the original image. mostly interesting is the fact that textural information has been cleared visible in an image which enhanced [3].

\section{An experimental psychologist law}

Weber- Fechner law is an experimental psychologist law where it is fine for different sensory scheme [2], which stated, "The ratio between the original intensity $I_{o}$ and the change in intensity $\left(\Delta I=I-I_{o}\right)$ is remains constant " $c$ "', $\left(\Delta I / I_{o}\right.$ $=c$ ). This ratio is called Weber's ratio, and $c$ is Weber's 


\section{International Journal of Science and Research (IJSR) \\ ISSN (Online): 2319-7064}

Index Copernicus Value (2015): 78.96 | Impact Factor (2015): 6.391

fraction, where $c$ for light intensity is, $c=.01 \ldots .02$. In this paper, according to this law, which is more sensitive to texture information, for each block, the block is classified as a background region if the ratio between $\Delta I$ (which is founded from the difference between the two means) and $I_{o}$ is smaller than a threshold value $c$. This paper presents a new image coding algorithm using the condition of this law which is an efficient method for decreasing computational complexity and improving compression results in low bit rates with good reconstructed enhanced image quality.

\section{Proposed method}

A Block Analysis is used to distinguish the background blocks of gray level image. In this paper, this technique is proposed to enhance the gray scale image, An enhancement and compression is implement using the information of block with modified Weber's law.

The first step proposed is block analysis where the input image is split into $n$ block of size $4 \times 4$ where each block is enhanced individually depending on Background analysis by block.

Let $G$ be the original image which is subdivided into $n$ blocks. For each block, the minimum intensity (low mean) $M l$ and maximum intensity (high mean) $M h$ values are calculated, these values are used to find the background criteria $\alpha$ by using equation 1 :

$$
\alpha_{i}=\frac{M l_{i}+M h_{i}}{2} \quad \forall i=1,2, \ldots \ldots, n
$$

Depending on the criteria value $\alpha$, where $\alpha$ is used as a threshold between dark and clear region. It is dark if $\boldsymbol{F}<=\boldsymbol{\alpha}$ and clear when $\boldsymbol{F}>\boldsymbol{\alpha}$. While the background parameter is totally dependent on the background criteria $\boldsymbol{\alpha}$ value, therefore, for $\boldsymbol{F}<=\boldsymbol{\alpha}$, the background parameter takes the maximum intensity value $M h$ within the analyzed block, otherwise takes the minimum intensity value $M l$ eq. 2 .

Study of contrast sensitivity of the human eye shows that our proposed method contrast sensitivity based on the Weber's Law, hence, we effectively apply this fact to design a contrast- enhancement method for images that has dark regions or poor lighting by controlling the information of each image block.

The next step encoding algorithm is examined. For each enhanced block, The proposed compression scheme makes use of bit plane coding, where the bit plan is determined. The bit plane coding divides the given image in to non overlapping blocks. Here, for each image block, according to the Weber-Fechner condition, if ( ( the difference between the higher and the lower means) / lower mean) is less than a threshold value ( Weber's fraction), the block is classified as low details block, then encode block with only the block mean $(\boldsymbol{M})$, and use "0" as an indicator bit as prefix code for decoding proposed then mean of block and " 0 " are transmitted. At the time of decoding bit plane omitted blocks are replaced by the respective block means. Otherwise the block is classified as high details block, then use "1" as an indicator bit as prefix code for decoding proposed and the two reconstruction level ( $\boldsymbol{M l}$ and $\boldsymbol{M h}$ ), the pit map with "1" are transmitted.

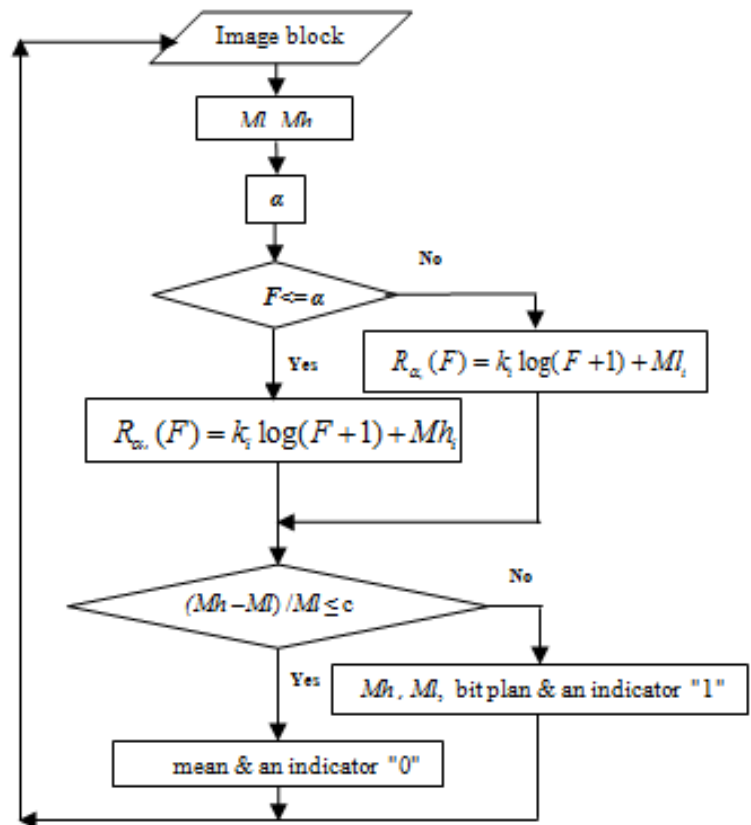

Figure 1: The detailed steps involved in an enhanced compression process

The interpolative technique is the method that drops partly of the bit plane at the time of encoding and decoding with enhanced of decoding image.

\section{Conclusion}

1) One can see that the enhanced image is more vision image than the original where the advanced of proposed method is the textural information has been rendered visible in the enhanced image see fig. $2, \mathrm{~b}$.

2) In the enhanced images, it can be seen that the objects that are dark visible region in the original image are appeared.

3) From the experimental results, utilizing block of size $4 x 4$, bit rate will be fall from $2 \mathrm{~b} / \mathrm{p}$ into $.49 \mathrm{~b} / \mathrm{p}$, with increasing in coding speed, low computation, high compression ratio, and good quality of reconstructed image, see table 1. all these can be achieved if the condition of WeberFechner law is applied on bit map when Weber's fraction $\beta$ is equal .02.

4) The experimental results show that the proposed schemes achieve good results, where the reconstructed of enhanced image quality obtained using proposed algorithm is good when compared it with other existing Btc method, see table 1 .

5) When the proposed algorithm based on Weber's law, one can see, the total calculation time at the transmitter is significantly reduced, it can be seen from table 2 that $61.163 \%$ bit map translation are eliminated by this process where only mean of block is transmitter, see table 2 . 


\section{International Journal of Science and Research (IJSR) \\ ISSN (Online): 2319-7064}

Index Copernicus Value (2015): 78.96 | Impact Factor (2015): 6.391

Table 1: PSNR with bit rate values for original Btc and proposed algorithm based on Weber-Fechner condition, the test image are $64 \times 64$ pixels.

\begin{tabular}{|l|l|l|l|l|l|l|}
\hline \multirow{2}{*}{$\begin{array}{l}\text { Block } \\
\text { size }\end{array}$} & \multirow{2}{*}{ Btc } & \multicolumn{3}{|c|}{$\begin{array}{l}\text { proposed algorithm based on } \\
\text { Weber-Fechner condition }\end{array}$} \\
\cline { 4 - 7 } & & \multicolumn{2}{|c|}{$\beta=.01$} & \multicolumn{1}{c|}{$\beta=.02$} \\
\cline { 3 - 7 } & B.r & PSNR & B.r & PSNR & B.r & PSNR \\
\hline $4 \times 4$ & 2 & 31.3 & .65 & 29.3 & .49 & 28.6 \\
\hline $8 \times 8$ & 1.25 & 28.1 & .52 & 27.2 & .31 & 26.1 \\
\hline
\end{tabular}

Table 2

\begin{tabular}{|c|c|}
\hline Block size & Reduce in bitmap transmitter \\
\hline $4 \times 4$ & $61.163 \%$ \\
\hline $8 \times 8$ & $47.206 \%$ \\
\hline
\end{tabular}

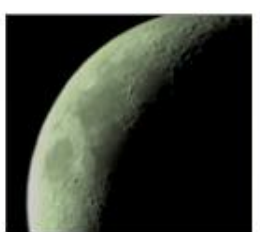

a.

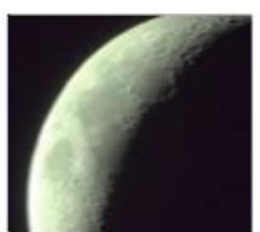

b.

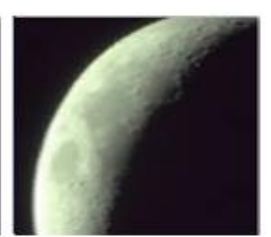

c.
Figure 2: Moon image, block size 4 x4

a. Original image..

b. Enhanced image .

c. An enhanced compressed image using Weber-Fechner condition $\mathrm{C}=.01$.

Finally the aim of this paper is to detect the background image and enhance the contrast in gray level image blocks which have poor or reduced lighting then compresses them in low bit rates with good quality.

\section{References}

[1] Sindhu M1, Rajkamal R2. "Images and Its Compression Techniques-A Review". International Journal of Recent Trends in Engineering, Vol. 2, No. 4, 2009.

[2] M. A. Hameed. "Digital Image Compression Using Adaptive Btc Method Based on Weber-Fechner's Measure". Al - Mustansiriya J. Sci, Vol. 23, No. 1, pp. 87-92, 2012.

[3] K. Sreedhar, B. Panlal. "ENHANCEMENT OF

IMAGESUSING MORPHOLOGICAL

TRANSFORMATIONS". International Journal of

Science \& Information Technology (IJCSIT), Vol. 4, No. 1, pp. 33-50, 2012.

[4] A. Vartak, V. Mankar. "Image Processing Techniques for Contrast Enhancement with Poor Lighting on Social and Medical Images". International Journal of Computers Applications, Vol. 123, No. 9, pp. 33-50, 2015.

[5] S. Sathya, R. Manavalan. "Analysis of Background Detection and Contrast Enhancement of MRI Images", International Journal of Computer Applications ( 09758887), Vol. 36, No.12, pp. 16-21, 2011.

[6] Y. - Gi Wu. "Block truncation image bit plane coding". Optical Engineering, Vol. 41, No. 10, pp. 2476-2478, 2002.

\section{Author Profile}

Maha Ahmed Hameed received the B.S. degree in Physics, University of Baghdad, College of Science, Dept. of Physics and M.S. degree in Image Processing in University of Baghdad, College of Science, Dept. of Astronomy in 1999. She is teaching in the Astronomy Dept. now.: 